rungsempfindlichkeit, auch Kopf- und Augenschmerzen sowie Motilitätsstörungen können vorkommen. Die Therapie erfolgt antibiotisch, ggf. chirurgisch mit Drainage der Siebbeinzellen von vorn.

\section{Enophthalmus}

Ein Enophthalmus, das Zurücksinken des Augapfels in die Orbita, entsteht insbesondere bei Blow-out-Fraktur der Orbita oder bei Atrophie von Orbitagewebe. Ein Pseudoenophthalmus findet sich beim Horner-Syndrom.

\section{Literatur}

Henderson J (1994) Orbital tumors. Raven Press, New York

Lommatsch PK (1999) Ophthalmologische Onkologie. Enke, Stuttgart

Nelson LB (ed) (1998) Harley's pediatric ophthalmology. Saunders, Philadelphia

Wright (1995) Pediatric ophthalmology and strabismus. Mosby, St. Louis

\title{
257 Erhöhter und erniedrigter Augeninnendruck
}

\section{T. Böker}

\section{Glaukom}

Das Glaukom (grüner Star) ist eine Krankheit mit kontinuierlichem Verlust von Nervenfasern des Sehnervs. Die Folgen sind eine zunehmende Exkavation des Sehnervenkopfes und progrediente Gesichtsfeldausfälle, die anfangs vom Patienten nicht bemerkt werden. Ein Hauptrisikofaktor ist die Erhöhung des Augeninnendrucks. Meist sind ältere Menschen betroffen, selten tritt ein Glaukom bei Neugeborenen und Kindern auf. Die ersten Zeichen sind dann vermehrter Tränenfluss, Photophobie, Blepharospasmus, Hornhauttrübung (Ödem) und zunehmende Vergrößerung des Auges (Buphthalmus) mit Zunahme des Hornhautdurchmessers. Papillenexkavation, Sehnervatrophie und Sehverlust können folgen.

Pathogenese. Das angeborene oder im frühen Kindesalter auftretende Glaukom ist üblicherweise Folge einer Fehlentwicklung des Kammerwinkels; meist findet man dort mesodermales Gewebe, das den Kammerwasserabfluss über das Trabekelmaschenwerk im Kammerwinkel blockiert. Das kongenitale Glaukom wird meist rezessiv vererbt, jedoch gibt es auch Formen einer dominant vererbten Kammerwinkeldysgenesie. Ein Glaukom kann mit anderen Krankheiten wie z. B. Aniridie, mesodermaler Dysgenesie, Sphärophakie, Neurofibromatose, Sturge-Weber-Syndrom, Lowe-Syndrom oder Marfan-Syndrom assoziiert sein. Zu Sekundärglaukomen kommt es nach Trauma, intraokularer Blutung, Uveitis, intraokularen Tumoren oder Kortisontherapie.

Therapie. Die Therapie des angeborenen oder im frühen Kindesalter auftretenden Glaukoms ist in der Regel chirurgisch.
Die Operation sollte möglichst schon in den ersten Lebenstagen erfolgen. Chirurgische Eingriffe, die der Druckregulation dienen, sind Goniotomie, Trabekulotomie, Trabekulektomie, tiefe Sklerektomie und zyklodestruktive Maßnahmen. Oft sind mehrere Eingriffe und eine begleitende lokale drucksenkende Therapie notwendig. Die Prognose der Sehschärfe hängt von der Drucknormalisierung und der Vermeidung von Sehnervenschäden ab. Begleitend müssen oft eine Korrektur von Refraktionsfehlern und eine Amblyopieprophylaxe mittels Okklusion durchgeführt werden. Gegebenenfalls assoziierte Veränderungen der Augen wie Katarakt, Hornhauttrübungen oder Krankheiten der Netzhaut müssen zusätzlich behandelt werden und beeinflussen die Prognose.

\section{Okuläre Hypotonie}

Eine okuläre Hypotonie kann Folge einer perforierenden Verletzung sein oder nach einer Uveitis auftreten. Eine akute Hypotonie tritt bei Kleinkindern mit mäßiger bis schwerer Dehydratation auf.

\section{Literatur}

Baez KA, Ulbig MW, Rice NSC (1994) Diagnostik und Therapie der frühkindlichen Glaukome. Ophthalmologe 91: 408

Draeger J (1993) Surgical measures in congenital glaucoma. Klin Monatsbl Augenheilkd 202: 425

McPherson SD Jr, Berry DP (1983) Goniotomy vs. external trabeculotomy for developmental glaucoma. Am J Ophthalmol 95: 427

Robin AL, Quigley HA, Pollack IP et al. (1979) An analysis of visual acuity, visual fields, and disc cupping in childhood glaucoma. Am J Ophthalmol 88: 847

\section{Verletzungen}

\section{P. Roggenkämper}

Die meisten Verletzungen verursachen Schmerzen, Lichtscheu, Tränen, Lidkrampf, Unterblutung oder Rötung der Haut und führen zur sofortigen Vorstellung bei einem Arzt. Ernsthafte Augenverletzungen gibt es jedoch auch ohne Vorliegen derartiger Symptome.
Für die meisten Augenverletzungen gilt: Es ist wichtiger, dass die Versorgung durch erfahrene Ärzte in einer gut ausgerüsteten Augenklinik erfolgt, als dass weite Anfahrtswege vermieden werden!

Lidschwellung, Rötung und Unterblutung der Lider sind häufig nach stumpfem Trauma zu beobachten. Diese Symp- 